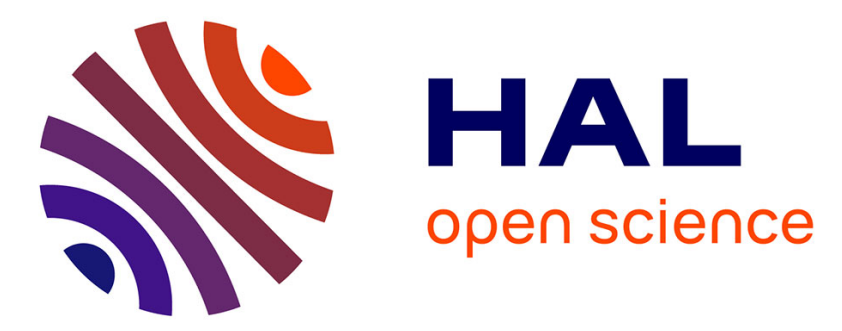

\title{
Lamin and the heart
}

Gabriella Captur, Eloisa Arbustini, Gisèle Bonne, Petros Syrris, Kevin Mills, Karim Wahbi, Saidi A Mohiddin, William J Mckenna, Stephen Pettit, Carolyn y Ho, et al.

\section{- To cite this version:}

Gabriella Captur, Eloisa Arbustini, Gisèle Bonne, Petros Syrris, Kevin Mills, et al.. Lamin and the heart. Heart, 2018, 104 (6), pp.468-479. 10.1136/heartjnl-2017-312338 . hal-03285171

\section{HAL Id: hal-03285171 \\ https://hal.sorbonne-universite.fr/hal-03285171}

Submitted on 13 Jul 2021

HAL is a multi-disciplinary open access archive for the deposit and dissemination of scientific research documents, whether they are published or not. The documents may come from teaching and research institutions in France or abroad, or from public or private research centers.
L'archive ouverte pluridisciplinaire HAL, est destinée au dépôt et à la diffusion de documents scientifiques de niveau recherche, publiés ou non, émanant des établissements d'enseignement et de recherche français ou étrangers, des laboratoires publics ou privés. 


\section{TITLE}

\section{Lamin and the Heart}

Gabriella Captur ${ }^{1,2,3}$ MD PhD MRCP MSc, Eloisa Arbustini MD, ${ }^{4}$ Gisèle Bonne $\mathrm{PhD},{ }^{5}$ Petros Syrris PhD ${ }^{6}$ Kevin Mills $\mathrm{PhD},{ }^{1,7}$ Karim Wahbi MD PhD ${ }^{5,8,9}$ Saidi A Mohiddin MBChB MD MRCP,${ }^{10}$ William J McKenna, ${ }^{6}$ Stephen Pettit MBBS PhD MRCP,${ }^{11}$ Carolyn Y Ho MD, ${ }^{12}$ Antoine Muchir PhD, ${ }^{5}$ Paul Gissen MBChB PhD MRCPCH, ${ }^{7,13,14}$ Perry M Elliott MBBS MD FRCP FESC FACC, ${ }^{2,3,6}$ James C Moon ${ }^{2,3,6}$ MD MBBS MRCP.

1. UCL Biological Mass Spectrometry Laboratory, Institute of Child Health and Great Ormond Street Hospital, 30 Guilford Street, London WC1N 1EH, UK

2. NIHR University College London Hospitals Biomedical Research Center, London, UK

3. Barts Heart Center, The Cardiovascular Magnetic Resonance Imaging Unit and The Inherited Cardiovascular Diseases Unit, St Bartholomew's Hospital, West Smithfield, London, UK

4. Center for Inherited Cardiovascular Diseases, Foundation IRCCS Policlinico San Matteo, University of Pavia, Pavia, Italy

5. Sorbonne Universités, UPMC Univ Paris 06, INSERM UMRS974, CNRS FRE3617, Centre de Recherche en Myologie, Institut de Myologie, G.H. Pitié Salpêtrière, Paris, France

6. Institute of Cardiovascular Science, University College London, Gower Street, London, WC1E 6BT, UK

7. Institute of Child Health, University College London, London, WC1N 1EH, UK

8. Sorbonne Paris Cité, Université Paris Descartes, Paris F-75006, France

9. AP-HP Groupe Hospitalier Cochin-Broca-Hôtel Dieu, Service de Cardiologie, Paris 75014, France

10. Barts Heart Center, William Harvey Research Institute, NIHR Cardiovascular Biomedical Research Unit at Barts, Queen Mary University of London, London, United Kingdom

11. Transplant Unit, Papworth Hospital NHS Foundation Trust, Cambridge, UK

12. Cardiovascular Division, Brigham and Women's Hospital, Boston MA, USA

13. MRC Laboratory for Molecular Cell Biology, University College London, London, WC1E 6BT, UK

14. Inherited Metabolic Diseases Unit, Great Ormond Street Hospital, London, WC1N 3JH, UK

\section{CORRESPONDENCE}

Prof James C Moon

Institute of Cardiovascular Science,

University College London,

Gower Street,

London WC1E 6BT, UK

E-mail: j.moon@ucl.ac.uk Phone No: +44 2034563081 Fax No: +0203 4563086

\section{FUNDING}

G.C. and J.C.M are supported by the National Institute for Health Research Rare Diseases Translational Research Collaboration (NIHR RD-TRC, \#171603), by the Society for Cardiovascular Magnetic Resonance (SCMR Seed Grant \#2017), and by University College London Hospitals NIHR Biomedical Research Center. J.C.M. is indirectly supported by the Biomedical Research Unit at Barts Hospital. P.S. is funded by the University College London Hospitals NIHR Biomedical Research Center and his research is supported by the Fondation Leducq. A.M, K.W. and G.B. are supported by the Institut National de la Santé et de la Recherche Médicale; the Université Pierre et Marie Curie-Paris 6, the Centre National de la Recherche Scientifique and the Association Française contre les Myopathies.

\section{COMPETING INTERESTS}

A.M. is a member of the scientific advisory board of AlloMek Therapeutics, LLC, a privately held pharmaceutical company developing small molecules targeting ERK1/2 signaling. All other authors have declared no conflicts of interest.

\section{CONTRIBUTORSHIP STATEMENT}

GC conducted the literature search, wrote the manuscript and made the figures and tables. JCM planned and wrote the manuscript and provided expert review of the manuscript. EA made a figure and provided expert review of the manuscript. PS conducted a literature search, reviewed a table and provided expert review of the manuscript. SP, GB, AM, KM, KW, SAM, WJM, CYH, PG and PME provided expert review of the manuscript.

ETHICS APPROVAL - Not applicable 


\begin{abstract}
Lamins $\mathrm{A}$ and $\mathrm{C}$ are intermediate filament nuclear envelope proteins encoded by the LMNA gene. Mutations in $L M N A$ cause autosomal dominant severe heart disease, accounting for $10 \%$ of dilated cardiomyopathy (DCM). Characterized by progressive conduction system disease, arrhythmia and systolic impairment, lamin $\mathrm{A} / \mathrm{C}$ heart disease is more malignant than other common DCMs due to high event rates even when the left ventricular impairment is mild. It has several phenotypic mimics but overall it is likely to be an underrecognized cause of DCM. In certain clinical scenarios, particularly familial DCM with early conduction disease, the pre-test probability of finding an LMNA mutation may be quite high.

Recognising lamin $\mathrm{A} / \mathrm{C}$ heart disease is important because implantable cardioverter defibrillators need to be implanted early. Promising oral drug therapies are within reach thanks to research into the mitogen-activated protein kinase (MAPK) and affiliated pathways. Personalised heart failure therapy may soon become feasible for $L M N A$, alongside personalised risk stratification, as variant-related differences in phenotype severity and clinical course are being steadily elucidated.

Genotyping and family screening are clinically important both to confirm and to exclude LMNA mutations, but it is the three-pronged integration of such genetic information with functional data from in vivo cardiomyocyte mechanics, and pathological data from microscopy of the nuclear envelope, that is properly reshaping our $L M N A$ knowledgebase, one variant at a time.

This review explains the biology of lamin A/C heart disease (genetics, structure and function of lamins), clinical presentation (diagnostic pointers, electrocardiographic and imaging features), aspects of screening and management, including current uncertainties, and future directions.
\end{abstract}




\section{INTRODUCTION}

Lamins $\mathrm{A}$ and $\mathrm{C}$ are encoded by the LMNA gene. LMNA mutations cause up to $10 \%$ of dilated cardiomyopathies (DCM) but they also underlie a spectrum of other diseases including some types of muscular dystrophy, lipodystrophy, and acrogeria syndromes (Table 1). In the heart, the spectrum ranges from no apparent cardiac disease, to isolated ventricular dilatation, arrhythmic cardiomyopathy, hypokinetic non-dilated cardiomyopathy, and overt DCM[1]. Accordingly we use the term "lamin A/C heart disease" (LHD). LHD is malignant causing heart failure, ventricular arrhythmias and cardiac conduction system disease (CCD) with an untreated sudden cardiac death (SCD) rate as high as 46\%[2]. Its earlier recognition is important because implantable cardioverter defibrillator therapy (ICD) can save lives. This review summarises the biology of LHD, its clinical presentation, aspects of screening and management, current uncertainties, and promising experimental therapies.

\section{WHAT ARE LAMINS}

Lamins are nuclear envelope proteins implicated in DNA replication, cell-cycle regulation, chromatin organization, differentiation maintenance, nuclear stability, pore positioning, gene expression and signal transduction. The lamin family comprises Type-A and Type-B Class V intermediate filaments. Type-B filaments (lamins B1, B2) are encoded by $2 L M N B$ genes, not known to cause cardiac disease. Type-A filaments derive from a single $L M N A$ gene on chromosome 1q21.2-21.3 (Figure 1A). LMNA undergoes alternative splicing of a single transcript generating 4 products, collectively known as 'lamin $\mathrm{A} / \mathrm{C}$ ' (see Figure 1 legend). Mutations within all exons and the first 10 introns of $L M N A$ cause cardiac disease (www.UMD.be/LMNA/, Supplementary Table 1 and Figure 2).

\section{MECHANISMS OF DISEASE}

The vast majority of disease causing $L M N A$ mutations are sequence-level alterations and are predominantly missense, but frameshift, nonsense, and intragenic deletions and duplications, capable of resulting in a lossof-function, are also reported. The clinical impact of LMNA mutations varies not only on the basis of molecular consequence (i.e. the non-missense vs. missense dichotomy alluded to in the European guideline- 
see Device section) but also on the basis of predicted amino acid change. For example, p.(Arg331Gln), p.(Arg190Trp) and p.(Glu161Lys) are all single nucleotide missense substitutions, but a patient with the former mutation is likely to exhibit a more benign course than another with either of the latter 2 (that are reportedly more malignant)[3]. Therefore LMNA mutation variant matters for clinical risk stratification due to differing effects on the structure and function of cardiomyocytes[3] resulting from a combination of pathophysiological processes (Figure 3). Possible mechanisms of pathogenicity include loss of LMNA function secondary to haploinsufficiency, but also dominant-negative or toxic gain-of-function mechanisms through mutations that alter heptad stability (Figure 1F) influencing dimerization. These patho-functionalgenetic effects are being systematically unravelled, not only through what we have learnt already from systemic laminopathy studies looking at nuclear mechanics and mechanotransduction in cultured embryonic fibroblasts from lamin $\mathrm{A} / \mathrm{C}$-deficient mice, but also from in vitro functional studies of human membranepermeabilized cardiomyocytes appraising force development and myofibril density. To understand the spectrum of variant-specific disease mechanisms in LHD, more studies are needed using this 3-pronged approach: integrating genetics, function and pathology (ultrastructural and immunofluorescent scrutiny of the nuclear envelope).

\section{DISEASE MANIFESTATIONS}

LHD is malignant in both males and females[4] although worse phenotypes and outcomes have been reported in males[5]. For the p.Arg225Ter nonsense variant for example, this gender difference was attributed to the nuclear accumulation of androgen receptor and its co-activators[6] but further research is needed.

Based on a small single site study, there was age-dependent penetrance and a major transition in phenotype development (from $7 \%$ to $66 \%$ ) at around 20 years[5] and complete penetrance by 60 years[5]. A typical untreated progression might be from premature atrial contractions to atrial arrhythmias, to $\mathrm{CCD}$, to hypokinetic non-dilated cardiomyopathy, to DCM but features may present "out-of-order" and in combination. 


\section{Conduction disease}

The most prominent and discriminatory cardiac feature of LHD is progressive CCD including sinoatrial and His/bundle disease[7] (Figure 4A-F) sometimes requiring pacing for sinoatrial dysfunction or high-grade atrioventricular block at a young age.

\section{Supraventricular and ventricular arrhythmias}

Supraventricular arrhythmias, particularly atrial fibrillation (AF) and flutter are common and may precede DCM development by decades unlike most DCM-related AF[2]. Premature ventricular complexes and nonsustained ventricular tachycardia (NSVT) are also common. Sustained VT and ventricular fibrillation (VF) cause SCD so LHD patients with pacemakers but not defibrillators, may still sustain SCD[8]. Malignant arrhythmias may occur in echocardiographically mild or undetectable disease[2], although rarely without at least some evidence of $\mathrm{CCD} /$ other supraventricular arrhythmias. The $L M N A$ mutation arrhythmia risk can also occur as part of a primarily non-cardiac or overlapped phenotype[2].

\section{Dilated cardiomyopathy}

LMNA mutations can cause isolated left ventricular (LV) enlargement, isolated LV dysfunction, or a typical DCM but marked LV dilatation and/or wall thinning is not characteristic[9]. Biventricular involvement can occur and be relevant for advanced heart failure care strategies like mechanical circulatory support[10]. As a genetic abnormality, LHD does not recur post transplantation.

\section{Thromboembolism}

Data from a single-site retrospective study have suggested a possible link between LMNA mutation carriage and arterial and venous thromboembolism even in non-manifesting carriers[11], but prospective multi-center verification is needed.

\section{DIAGNOSTICS}

\section{Initial clinical workup}

In suspected LHD, particular features to seek include a 3 to 4 generation family history for familial DCM and $\mathrm{CCD}[1]$, sudden unexplained death, transplantation, AF, early pacemaker implantation, and Page of $\mathbf{3 4}$ 
neuromuscular disease. First-level investigations should include ECG and 24-hour Holter. Echocardiography reports should emphasise and not dismiss borderline/mild dilatation and/or impairment. Basic blood investigations should include serum creatinine phosphokinase (CK). Diagnostic 'red flags' (Table 2) should trigger a second-level work-up including further blood biomarkers, advanced imaging and genetic testing. When there is high diagnostic suspicion or a confirmed diagnosis of LHD, review for neuromuscular, endocrine and metabolic symptoms and/or onward referral to neurology may be appropriate. Conversely, non-cardiac-predominant laminopathies should have a basic cardiac work-up with low threshold for referral to cardiology.

\section{Imaging}

Echocardiography is a first-level investigation to assess biventricular size and function, regional wall motion and atrial size, while excluding alternative diagnoses. Diastolic dysfunction occurs in LHD irrespective of systolic dysfunction, correlating with left atrial dilatation and midmyocardial fibrosis by cardiovascular magnetic resonance (CMR)[12]. Septal speckle tracking longitudinal strain is reduced, correlating with PR interval prolongation[13] and predicting ventricular arrhythmias. Mechanical dispersion by strain is increased in LMNA carriers with NSVT and VT irrespective of ejection fraction and global longitudinal strain, and may be an early ventricular arrhythmia marker[14]. A thick layer of right ventricular epicardial fat may indicate associated familial partial lipodystrophy. CMR permits accurate volume assessment, with better repeatability compared to unenhanced transthoracic echocardiography[15], which is key to detecting subtle drops in LV function when following up mutation positive carriers. CMR basal to mid septal midmyocardial late gadolinium enhancement appears to be a common and early finding (Figure 4G-L)[9], correlating with $\mathrm{CCD}$ and ventricular arrhythmias, and matching the scar pattern observed at autopsy[12]. Extensive scar may uncommonly be found in advanced disease, although most at this stage have devices. The extracellular volume fraction may be elevated early[16].

\section{Blood biomarkers}

There are currently no clinically available specific LHD blood biomarkers. Serum CK levels may detect associated skeletal myopathy increasing the likelihood of LHD and justifying genetic testing in sporadic DCM[1] but a normal CK does not exclude the condition. N-terminal pro-B-type natriuretic peptide is 
elevated in LHD with LV dilatation/impairment but also in preserved LV function where it correlates with ventricular arrhythmias[16]. Aldosterone is at the upper limits of normal and dyslipidaemia common (a possible metabolic complication linked to lipodystrophy).

\section{Biopsy and autopsy}

Light and electron microscopy findings in LHD are summarised in Figure 5 and Table 3. Conventional light microscopy in advanced disease reveals extensive cardiomyocyte and conduction tissue abnormalities but these are non-specific. The nuclear ultrastructure requires electron microscopy and published results vary with one study suggesting preserved nuclear membrane integrity[17] but most others describing potentially pathognomonic disruptions of the nuclear membrane structure. Myocardial biopsy may help exclude other diseases such as sarcoid, myocarditis, arrhythmogenic cardiomyopathy and amyloidosis. Histology of autopsied hearts has confirmed basal septal midmyocardial fibrosis, matching that by CMR, in $75 \%$ of deceased LHD patients[12].

\section{Genetics}

The frequency of $L M N A$ mutations is up to $5 \%$ in patients with sporadic idiopathic DCM[18], $5 \%-10 \%$ in idiopathic familial DCM[19], and 33\% in familial DCM with CCD[20] (Table 2). Genetic testing should be considered for an index case who has: DCM and $\operatorname{CCD}\left(1^{\text {st }}, 2^{\text {nd }}\right.$ or $3^{\text {rd }}$ degree atrioventricular block $)$ and/or a family history of premature unexpected sudden death (Class I recommendation); familial DCM (Class IIa recommendation); isolated CCD especially when there is a family history of CCD (Class IIb recommendation); survived an unexplained out-of-hospital cardiac arrest if the medical evaluation raises a suspicion of genetic cardiomyopathy (Class I recommendation)[21]. For DCM not caused by mutations in $L M N A$, identifying a genetic cause in a proband is often of limited importance for the individual but more relevant to the family in terms of cascade screening and family planning[21]. Not so for LMNA mutations, given the higher prevalence of SCD, cardiac transplantation, and ventricular arrhythmias compared to DCM caused by sarcomere gene mutations[22], the consequently lower threshold for prophylactic ICD implantation,[23] and because therefore, the exclusion of an LMNA mutation is in itself reassuring[21].

Compared to some other forms of DCM, genetic testing in LHD may be potentially more straight forward as the LMNA gene is relatively small, the disease principally autosomal dominant and heterozygous (except for 
rare highly malignant homozygous disease or digenism e.g. $L M N A$ plus emerin), and its mutations usually exonic (80\%) single nucleotide substitutions with predictable functional consequences. LMNA gene testing can be performed in isolation (e.g. a DCM-CCD proband with high pre-test probability) but more often as part of a multi-gene DCM panel. Other gene mutations potentially mimic LHD (Table 4) and there may be value in identifying these where funding allows. Even with next-generation sequencing and larger panels the sensitivity for detecting the DCM-causing mutation may only reach $50 \%$. More is not necessarily better as broad-based sequencing approaches (Supplementary Table 2) may generate uncertainty by uncovering more novel variants of uncertain significance[24]. Judging the relevance of such novel variations can be helped by screening families (see next section) so that with time, information on most of the mutations coupled with data from mechanism studies, will become available in databases linking to clinical phenotypes. Given the significant phenotypic pleiotropy observed in spite of mutational invariance across and within families with $L M N A$ mutations, there is a risk for selection bias causing over-representation of the more severe LHD presentations in the published literature. Reports on milder and variable phenotypes such as that arising from the founder mutation p.(Arg331Gln)[22], are therefore important.

\section{Family screening}

Family screening for $L M N A$ occurs typically for family members of a living or deceased proband with a known pathogenic $L M N A$ mutation[25] where it is a Class I recommendation[21] or for relatives of a sudden death victim of unknown genetic status (Class IIa | level of recommendation C[25]). First degree relatives of a living DCM proband with suspected but unconfirmed LHD warrant echocardiography and ECG beyond 10-12 years of age, and if CCD is also a feature, 24-hour Holter[26]. Genotype-positive relatives are to be offered clinical surveillance while genotype-negatives can be reassured and discharged from follow up[27]. No cost-benefit assessment has yet appraised whether family screening by a combined genetic test and clinical screening approach is superior to sole clinical screening in LHD[28]. The number of asymptomatic relatives identified with $L M N A$ mutations and subclinical phenotypes is increasing[1] but data concerning surveillance intensity remains sparse though we suggest annual follow-up (history, ECG, Holter, echocardiography) seeking CCD/arrhythmia and LV dilatation and/or impairment, as risk markers for increasing arrhythmia risk. 


\section{CLINICAL MANAGEMENT-CURRENT PRACTICES}

\section{Lifestyle advice}

In the absence of cardiac implantable electronic devices, there is only limited data currently to support the discontinuation of high dynamic competitive sport in manifesting and non-manifesting carriers[5]-further validation of findings is needed prior to the wider acceptance of such a recommendation. The other common question that arises is regarding the safety of child-bearing in females with $L M N A$ mutations. Again, there is insufficient published data to understand true risk. In one small, retrospective case report that overrepresented a single mutation among 5 women with early clinical disease and no heart failure, pregnancy was well tolerated[29] but of course larger studies are needed.

\section{Conventional drug therapy}

The benefits of conventional heart failure therapies are unknown in LHD, except for case reports, animal studies and one phase III clinical trial (PRECARDIA, NCT01583114) prematurely stopped due to poor recruitment. There is consensus for their use in LHD patients with LV dysfunction and heart failure; less so in non-manifesting carriers and early phenotype patients. Beta-blockers may improve symptoms, prognosis and reduce the risk of ventricular arrhythmias, but there is a risk of high-grade atrioventricular block in those with $\mathrm{CCD}$ and no pacemaker, and of increased right ventricular pacing in those without a cardiac resynchronisation therapy device[30]. Furthermore, metoprolol-treated LMNA mouse models developed heart failure, and more fibrosis compared to untreated mice[31]. The LMNA-related prothrombotic state needs further support in larger studies but if confirmed, would suggest a lower threshold for prophylaxis with anticoagulant or antiplatelet therapy-currently the evidence is missing.

\section{Device therapy}

LHD poses a greater risk of SCD than other DCMs, so early implantation of a primary prevention ICD may be warranted[32]. Its progressive nature and associated CCD also suggest a role for cardiac resynchronisation therapy. Symptomatic sinoatrial disease or high-grade atrioventricular block mean many have an indication for pacing but, SCD from malignant arrhythmias may still occur[33]. Where an ICD is implanted in the presence of a traditional pacing-only indication and a pathogenic LMNA mutation, 
appropriate ICD therapy rates are high ( $42 \%$ within 3 years and $52 \%$ within 5 years, $n=40$ total patients in 2 studies[32,34]).

$L M N A$ is one of the first named genes to be included in SCD risk stratification algorithms. Primary prevention ICD implantation can be useful in LMNA-positive patients with progressive CCD and LV dysfunction or NSVT (Class IIa; level of evidence C[35]) and in LMNA-positive DCM with 'clinical risk factors' (Class IIa; level of evidence B[25]) these being, NSVT, LV ejection fraction $<45 \%$, male gender and non-missense mutations. Risk factors are based on a retrospective multi-center study of 269 LHD patients[36] where malignant ventricular arrhythmias were only observed in those with at least 2 risk factors, with each additional risk factor inflating the risk[36]. Male gender, non-missense mutations and left ventricular ejection fraction $\leq 50 \%$ similarly predicted malignant ventricular arrhythmias in a separate, more recent retrospective outcome study $(n=122)$, but importantly, malignant ventricular arrhythmias also occurred in those with 0 or 1 risk factors[37]. Other candidate risk factors include poor functional capacity[5], > 10 years exposure to competitive sports[5], basal septal scar by CMR[13], PR interval prolongation[13], and mechanical dispersion by strain[14]. Important questions remain: is every young male with a non-missense $L M N A$ mutation expected to receive an ICD?; are the 4 major clinical risk factors of equal importance? what additional risk is conferred by the other published risk factors?; and could some specific missense mutations (e.g. p.Arg190Trp) be about as malignant as truncation-predicting ones?

There are potential adverse implications of implanting an earlier lifesaving ICD rather than a pacemaker. ICD pulse generators are larger with shorter battery longevity requiring potentially several pulse generator replacements within the lifespan of a young lamin patient resulting in a significant lifetime risk of devicerelated complications[38]. A recent LHD meta-analysis suggests annual rates of appropriate ICD therapy of $5.6 \%$ and $17.1 \%$ for primary and secondary prevention ICDs respectively, but also annual rates of inappropriate shocks and total device-related complication of $4.6 \%$ and $10.3 \%$, including very rare fatalities[39].

\section{Electrophysiological studies and catheter ablation}

The early potential of electrophysiology study inducing VT or VF for risk stratification[2] may not be borne out in LHD where the VT substrate is more complex. 
There are also doubts about the effectiveness of catheter ablation for VT in LHD where the combination of a predominantly intramural VT substrate and basal perivalvular scar make VT ablation particularly challenging[40] and may not prevent arrhythmia recurrence or progression to advanced heart failure[40]. Catheter ablation of clinical VT in non-ischaemic DCM is therefore reserved for patients presenting with a clear VT mechanism (e.g. bundle branch re-entry) and only in experienced centers.

\section{Heart transplantation}

Patients with LHD who develop advanced heart failure or recurrent ventricular arrhythmias may be candidates for heart transplantation[41]. During assessment, extra-cardiac manifestations such as skeletal muscle involvement should be considered because of the risk of muscle toxicity from calcineurin inhibitors, post-operative deterioration of muscle symptoms, and statin intolerance. Selected patients may undergo mechanical circulatory support as a bridge to heart transplantation if required.

\section{FUTURE BIOMARKERS}

Proteomics-based clinical tests for LHD are in early phase. Skin fibroblasts in LMNA mutation carriers differentially express proteins implicated in, or downstream of, pathways elaborated in Figure 3[42]. A targeted metabolomics assay has been developed using heart extracts from a homozygous LMNA knock-out mouse model with aggressive multisystem disease resulting from complete loss of lamin function[43]. However, the null model's faithfulness to human LHD is unknown and likewise the assay's applicability to the human condition.

Patients with LHD have reduced circulating blood LMNA mRNA compared to DCM not caused by mutations in $L M N A$, and controls[44]. The reduction mirrors nuclear membrane lamin under-expression in immunostained myocardial biopsy samples from the same patients. The extent of mRNA under-expression did not correlate with mutation type or position on cDNA relative to the nuclear localization signal, but this may partly be due to an exposure-response relationship determining transcript abundance. It has been shown that transcript abundance decreased over time in LHD patients, suggesting that single time point mRNA measurements might be influenced by the patients' age and/or time since phenotypic penetrance. This lowcost transcriptomic biomarker needs further refinement, but could potentially gain a clinically useful role as a 
pre-genetic assay to guide genetic analysis and to monitor disease progression and treatment response in LHD patients[44].

\section{FUTURE THERAPIES}

Cardiac mitogen-activated protein kinases (MAPKs) and protein kinase B (AKT)/mammalian target of rapamycin (mTOR) signaling pathways are very complex signaling systems that have been shown to be activated prior to overt LHD (Figures 3B,C) in a knock-in Lmna ${ }^{H 222 P}$ mouse model (p.His222Pro), in mice deficient of lamin A/C (Lmna $\left.{ }^{-/}\right)[45]$, and in explanted human LHD myocardium. At least 3 MAPK and AKT/mTOR pathway inhibitors have been investigated: 1) inhibitor of MEK1/2 (the MAPK kinase that phosphorylates ERK1/2 early along the MAPK pathway); 2) inhibitor of c-Jun N-terminal kinases (JNK); and 3) inhibitor of p38 $\alpha$ (Figure 3B), with MEK1/2 inhibitors being furthest along clinical development.

The first small molecule allosteric inhibitor of MEK1/2 to be tested (PD98059, Pfizer) improved LV size and function in mice[46] but was superseded by another more potent and selective inhibitor, Selumetinib (Array BioPharma), now in phase III clinical trials for cancer. Another such allosteric, macrocyclic MEK1/2 inhibitor (Allomek Therapeutics LLC) was successfully used in Lmna ${ }^{H 222 P}$ mice. The reversible JNK inhibitor SP600125 (Calbiochem) tested in Lmna ${ }^{H 222 P}$ mice prevented LV dilatation, improved systolic function and decreased myocardial fibrosis but is early phase. A small molecule inhibitor of p38 (ARRY371797, Array BioPharma) used in Lmna ${ }^{H 222 P}$ mice, improved left ventricular diameters and fractional shortening and has now completed phase II clinical trial. Results are keenly awaited as some of these promising therapies may have negative side effects when applied to humans. The pyridazinone derivative calcium sensitizer SCH00013, tested in $\mathrm{Lmna}^{H 222 P}$ mice, improves myocardial contractility and reduces myocardial fibrosis with an overall prosurvival effect and is also early phase[47].

The mutated LMNA cell-signaling environment antagonizes autophagy so pharmacological reversal could work. Modulation of the Akt/mTOR pathway by temsirolimus (Figure 3C) in mouse models restores autophagy[48] ameliorating cardiac function.

Where toxic lamin accumulation is suspected, an antisense oligonucleotide strategy targeting exon 11, may treat progeria by reducing prelamin A and tipping the balance of splicing towards lamin C. This approach may be applicable LHD caused by missense mutations in exon 11[49]. 


\section{CONCLUSION}

Lamin $\mathrm{A} / \mathrm{C}$ heart disease is an important cause of DCM, particularly common with conduction disease, but the presentation can be diverse, to different cardiology and medical specialities. Device therapy is important and instituted early with specific risk stratification. The clinician should be alert to LHD and its diagnostic 'red flags', the mild echocardiographic phenotype early on, the conduction system predilection, ventricular arrhythmia risk and multisystem involvement. Genotyping adds major value, both to individuals and their families, and, with coordinated clinical and basic research at scale, new biomarkers and emerging diseasespecific therapies have the potential to halt or even reverse the nuclear devastation that underpins lamin $\mathrm{A} / \mathrm{C}$ heart disease.

\section{REFERENCES}

\section{Bibliography\}}

1 Pinto YM, Elliott PM, Arbustini E, et al. Proposal for a revised definition of dilated cardiomyopathy, hypokinetic non-dilated cardiomyopathy, and its implications for clinical practice: A position statement of the ESC working group on myocardial and pericardial diseases. Eur Heart J 2016;37:1850-8. doi:10.1093/eurheartj/ehv727

2 Van Berlo JH, De Voogt WG, Van Der Kooi AJ, et al. Meta-analysis of clinical characteristics of 299 carriers of LMNA gene mutations: Do lamin A/C mutations portend a high risk of sudden death? J Mol Med 2005;83:79-83. doi:10.1007/s00109-004-0589-1

3 Arbustini E, Favalli V, Narula N. LMNA mutations associated with mild and late-onset phenotype: The case of the Dutch founder mutation p.(Arg331Gln). Circ Cardiovasc Genet 2017;10:e001816. doi:10.1161/CIRCGENETICS.117.001816

4 Van Rijsingen IAW, Nannenberg EA, Arbustini E, et al. Gender-specific differences in major cardiac events and mortality in lamin A/C mutation carriers. Eur J Heart Fail 2013;15:376-84. doi:10.1093/eurjhf/hfs191

5 Pasotti M, Klersy C, Pilotto A, et al. Long-term outcome and risk stratification in dilated cardiolaminopathies. J Am Coll Cardiol 2008;52:1250-60. doi:10.1016/j.jacc.2008.06.044

6 Arimura T, Onoue K, Takahashi-Tanaka Y, et al. Nuclear accumulation of androgen receptor in gender difference of dilated cardiomyopathy due to lamin $\mathrm{A} / \mathrm{C}$ mutations. Cardiovasc Res 2013;99:382-94. doi:10.1093/cvr/cvt106

7 Brodt C, Siegfried JD, Hofmeyer M, et al. Temporal relationship of conduction system disease and ventricular dysfunction in LMNA cardiomyopathy. J Card Fail 2014;19:233-9. doi:10.1016/j.cardfail.2013.03.001.Temporal

8 Van Berlo JH, Duboc D, Pinto YM. Often seen but rarely recognised: Cardiac complications of lamin A/C mutations. Eur Heart J 2004;25:812-4. doi:10.1016/j.ehj.2004.03.007 
9 Holmström M, Kivistö S, Heliö T, et al. Late gadolinium enhanced cardiovascular magnetic resonance of lamin $\mathrm{A} / \mathrm{C}$ gene mutation related dilated cardiomyopathy. $J$ Cardiovasc Magn Reson 2011;13:30. doi:10.1186/1532-429X-13-30

10 Argiriou M, Kolokotron S-M, Sakellaridis T, et al. Right heart failure post left ventricular assist device implantation. J Thorac Dis 2014;6:S52-9. doi:10.3978/j.issn.20721439.2013.10.26

11 Van Rijsingen IAW, Bakker A, Azim D, et al. Lamin A/C mutation is independently associated with an increased risk of arterial and venous thromboembolic complications. Int $J$ Cardiol 2013;168:472-7. doi:10.1016/j.ijcard.2012.09.118

12 Raman S V, Sparks EA, Baker PM, et al. Mid-myocardial fibrosis by cardiac magnetic resonance in patients with lamin A/C cardiomyopathy: possible substrate for diastolic dysfunction. J Cardiovasc Magn Reson 2007;9:907-13. doi:10.1080/10976640701693733

13 Hasselberg NE, Edvardsen T, Petri H, et al. Risk prediction of ventricular arrhythmias and myocardial function in Lamin A/C mutation positive subjects. Europace 2014;16:563-71. doi:10.1093/europace/eut291

14 Haugaa KH, Hasselberg NE, Edvardsen T. Mechanical dispersion by strain echocardiography: A predictor of ventricular arrhythmias in subjects with lamin A/C mutations. JACC Cardiovasc Imaging 2015;8:104-6. doi:10.1016/j.jcmg.2014.04.029

15 Hoffmann R, Barletta G, Von Bardeleben S, et al. Analysis of left ventricular volumes and function: A multicenter comparison of cardiac magnetic resonance imaging, cine ventriculography, and unenhanced and contrast-enhanced two-dimensional and threedimensional echocardiography. J Am Soc Echocardiogr 2014;27:292-301. doi:10.1016/j.echo.2013.12.005

16 Barison A, Fontana M, Botto N, et al. CMR-verified interstitial myocardial fibrosis as a marker of subclinical cardiac involvement in LMNA mutation carriers. JACC Cardiovasc Imaging 2013;6:124-6. doi:10.1016/j.jcmg.2012.06.013

17 Otomo J, Kure S, Shiba T, et al. Electrophysiological and histopathological characteristics of progressive atrioventricular block accompanied by familial dilated cardiomyopathy caused by a novel mutation of lamin A/C gene. J Cardiovasc Electrophysiol 2005;16:137-45. doi:10.1046/j.1540-8167.2004.40096.x

18 Millat G, Bouvagnet $\mathrm{P}$, Chevalier $\mathrm{P}$, et al. Clinical and mutational spectrum in a cohort of 105 unrelated patients with dilated cardiomyopathy. Eur J Med Genet 2011;54:e570-5. doi:10.1016/j.ejmg.2011.07.005

19 Arbustini E, Pilotto A, Repetto A, et al. Autosomal dominant dilated cardiomyopathy with atrioventricular block: A lamin A/C defect-related disease. J Am Coll Cardiol 2002;39:98190. doi:10.1016/S0735-1097(02)01724-2

20 Kärkkäinen S, Heliö T, Miettinen R, et al. A novel mutation, Ser143Pro, in the lamin A/C gene is common in finnish patients with familial dilated cardiomyopathy. Eur Heart $J$ 2004;25:885-93. doi:10.1016/j.ehj.2004.01.020 
21 Ackerman MJ, Priori SG, Willems S, et al. HRS/EHRA Expert consensus statement on the state of genetic testing for the channelopathies and cardiomyopathies. Europace 2011;13:1077-109. doi:10.1093/europace/eur245

22 Kayvanpour E, Sedaghat-Hamedani F, Amr A, et al. Genotype-phenotype associations in dilated cardiomyopathy: meta-analysis on more than 8000 individuals. Clin Res Cardiol 2017;106:127-39. doi:10.1007/s00392-016-1033-6

23 Japp AG, Gulati A, Cook SA, et al. The diagnosis and evaluation of dilated cardiomyopathy. J Am Coll Cardiol 2016;67:2996-3010. doi:10.1016/j.jacc.2016.03.590

24 McNally EM, Puckelwartz MJ. Genetic variation in cardiomyopathy and cardiovascular disorders. Circ J 2015;79:1409-15. doi:10.1253/circj.CJ-15-0536

25 Priori S, Blomström-Lundqvist C, Mazzanti A, et al. 2015 ESC Guidelines for the management of patients with ventricular arrhythmias and the prevention of sudden cardiac death. Eur Heart J 2015;8:746-837. doi:10.1093/europace/eul108

26 Charron P, Arad M, Arbustini E, et al. Genetic counselling and testing in cardiomyopathies: a position statement of the European Society of Cardiology Working Group on Myocardial and Pericardial Diseases. Eur Heart J 2010;31:2715-26. doi:10.1093/eurheartj/ehq271

27 Mogensen J, Van Tintelen JP, Fokstuen S, et al. The current role of next-generation DNA sequencing in routine care of patients with hereditary cardiovascular conditions: a viewpoint paper of the European Society of Cardiology working group on myocardial and pericardial diseases and members of the European Society of Human Genetics. Eur Heart $J$ 2015;36:1367-70. doi:10.1093/eurheartj/ehv122

28 Wordsworth S, Leal J, Blair E, et al. DNA testing for hypertrophic cardiomyopathy: a costeffectiveness model. Eur Heart J 2010;31:926-35. doi:10.1093/eurheartj/ehq067

29 Palojoki E, Kaartinen M, Kaaja R, et al. Pregnancy and childbirth in carriers of the lamin A/C-gene mutation. Eur J Heart Fail 2010;12:630-3. doi:10.1093/eurjhf/hfq059

30 Wilkoff BL, Cook JR, Epstein AE, et al. Dual-chamber pacing or ventricular backup pacing in patients with an implantable defibrillator: the Dual Chamber and VVI Implantable Defibrillator (DAVID) Trial. JAMA 2002;288:3115-23.

31 Beqqali A, van Rijsingen I, van der Made I, et al. Abstract 156: Enalapril prevents development of dilated cardiomyopathy in lamin A/C mutant mice. Circ Res 2015;113.

32 Meune $\mathrm{C}$, Van Berlo JH, Anselme F, et al. Primary prevention of sudden death in patients with Lamin A/C gene mutations. N Engl J Med 2006;354:209-10. doi:10.1056/NEJMc052632

33 Wolf CM, Wang L, Alcalai R, et al. Lamin A/C haploinsufficiency causes dilated cardiomyopathy and apoptosis-triggered cardiac conduction system disease. J Mol Cell Cardiol 2008;44:293-303. doi:10.1016/j.yjmcc.2007.11.008

34 Anselme F, Moubarak G, Savouré A, et al. Implantable cardioverter-defibrillators in lamin A/C mutation carriers with cardiac conduction disorders. Hear Rhythm 2013;10:1492-8. 
doi:10.1016/j.hrthm.2013.06.020

35 Priori SG, Wilde AA, Horie M, et al. Executive summary: HRS/EHRA/APHRS expert consensus statement on the diagnosis and management of patients with inherited primary arrhythmia syndromes. Europace 2013;15:1389-406. doi:10.1093/europace/eut272

36 Van Rijsingen IAW, Arbustini E, Elliott PM, et al. Risk factors for malignant ventricular arrhythmias in Lamin A/C mutation carriers: A European cohort study. J Am Coll Cardiol 2012;59:493-500. doi:10.1016/j.jacc.2011.08.078

37 Kumar S, Baldinger SH, Gandjbakhch E, et al. Long-term arrhythmic and nonarrhythmic outcomes of lamin A/C mutation carriers. J Am Coll Cardiol 2016;68:2299-307. doi:10.1016/j.jacc.2016.08.058

38 Kirkfeldt RE, Johansen JB, Nohr EA, et al. Complications after cardiac implantable electronic device implantations: An analysis of a complete, nationwide cohort in Denmark. Eur Heart J 2014;35:1186-94. doi:10.1093/eurheartj/eht511

39 Olde Nordkamp LRA, Postema PG, Knops RE, et al. Implantable cardioverter-defibrillator harm in young patients with inherited arrhythmia syndromes: A systematic review and metaanalysis of inappropriate shocks and complications. Hear Rhythm 2016;13:443-54. doi:10.1016/j.hrthm.2015.09.010

40 Kumar S, Androulakis AFA, Sellal J-M, et al. Multicenter experience with catheter ablation for ventricular tachycardia in Lamin A/C cardiomyopathy. Circ Arrhythmia Electrophysiol 2016;9:e004357. doi:10.1161/CIRCEP.116.004357

41 Karkkainen S, Reissell E, Helio T, et al. Novel mutations in the lamin A/C gene in heart transplant recipients with end stage dilated cardiomyopathy. Heart 2005;92:524-6. doi:10.1136/hrt.2004.056721

42 Magagnotti C, Bachi A, Zerbini G, et al. Protein profiling reveals energy metabolism and cytoskeletal protein alterations in LMNA mutation carriers. Biochim Biophys Acta - Mol Basis Dis 2012;1822:970-9. doi:10.1016/j.bbadis.2012.01.014

43 West JA, Beqqali A, Ament Z, et al. A targeted metabolomics assay for cardiac metabolism and demonstration using a mouse model of dilated cardiomyopathy. Metabolomics 2016;12:1-18. doi:10.1007/s11306-016-0956-2

44 Narula N, Favalli V, Tarantino P, et al. Quantitative expression of the mutated lamin a/c gene in patients with cardiolaminopathy. J Am Coll Cardiol 2012;60:1916-20. doi:10.1016/j.jacc.2012.05.059

45 Muchir A, Wu W, Choi JC, et al. Abnormal p38 a mitogen-activated protein kinase signaling in dilated cardiomyopathy caused by lamin A/C gene mutation. Hum Mol Genet 2012;21:4325-33. doi:10.1093/hmg/dds265

46 Muchir A, Shan J, Bonne G, et al. Inhibition of extracellular signal-regulated kinase signaling to prevent cardiomyopathy caused by mutation in the gene encoding A-type lamins. Hum Mol Genet 2009;18:241-7. doi:10.1093/hmg/ddn343 
47 Arimura T, Sato R, Machida N, et al. Improvement of left ventricular dysfunction and of survival prognosis of dilated cardiomyopathy by administration of calcium sensitizer SCH00013 in a mouse model. J Am Coll Cardiol 2010;55:1503-5. doi:10.1016/j.jacc.2009.10.065

48 Choi JC, Worman HJ. Reactivation of autophagy ameliorates LMNA cardiomyopathy. Autophagy 2013;9:110-1. doi:10.4161/auto.22403

49 Lee JM, Nobumori C, Tu Y, et al. Modulation of LMNA splicing as a strategy to treat prelamin A diseases. J Clin Invest 2016;126:1592-602. doi:10.1172/JCI85908 


\section{FIGURES}

Figure 1. Schema showing biogenesis of wildtype lamin protein, from gene to higher-order assembly into lamin filaments.

The LMNA gene (A) uses an alternative 5' splice site in intron 10 to generate 4 Type-A proteins: lamin A (major isoform, B), A $\Delta 10$ (minor isoform, missing exon 10), C (known as C1, C) and C2 (only in germ cells). Each has a tripartite domain organisation: short globular head domain; central $\alpha$-helical rod domain; and immunoglobulin-like globular tail domain. Exon 1 codes for the head domain and first portion of the rod domain, the remainder encoded by exons 2-6. Exons 7-9 code for tail domain sequences common to both lamins A and C. These are identical for their first 566 a.a. but have distinct tail domains. Prelamin A (D) is the lamin A precursor with 98 unique $C$-terminal a.a. The -CaaX motif is post-translationally farnesylated, cleaved, methylated and cleaved again forming mature lamin A. Lamin A tail domain is encoded by part of exon 10 and the entire exons 11 and 12. Lamin C tail domain is encoded by sole exon 10, has 4 unique a.a, lacks a -CaaX box sequence and is not further modified after synthesis. Two parallel lamin monomers form lamin hetero/homodimers (E) without stagger, through coiled-coil interaction of heptad repeats (F) in the central rod domains. Inner hydrophobic and electrostatic residues in the heptad (green) stabilize its secondary structure, while charged a.a. pointing outwards (pink) affect higher-order assembly or intermolecular interactions. Dimers form polar "head-to-tail" assemblies (G) by overlapping their terminal rod domains. These form non-polar protofilaments composed of two antiparallel polymers $(\mathbf{H})$ that laterally assemble to form thick filament bundles, tangles and paracrystalline arrays.

a.a., amino acid; -CaaX, a four-amino acid sequence at the carboxyl terminus of a protein in which ' $a$ ' refers to an aliphatic residue. 


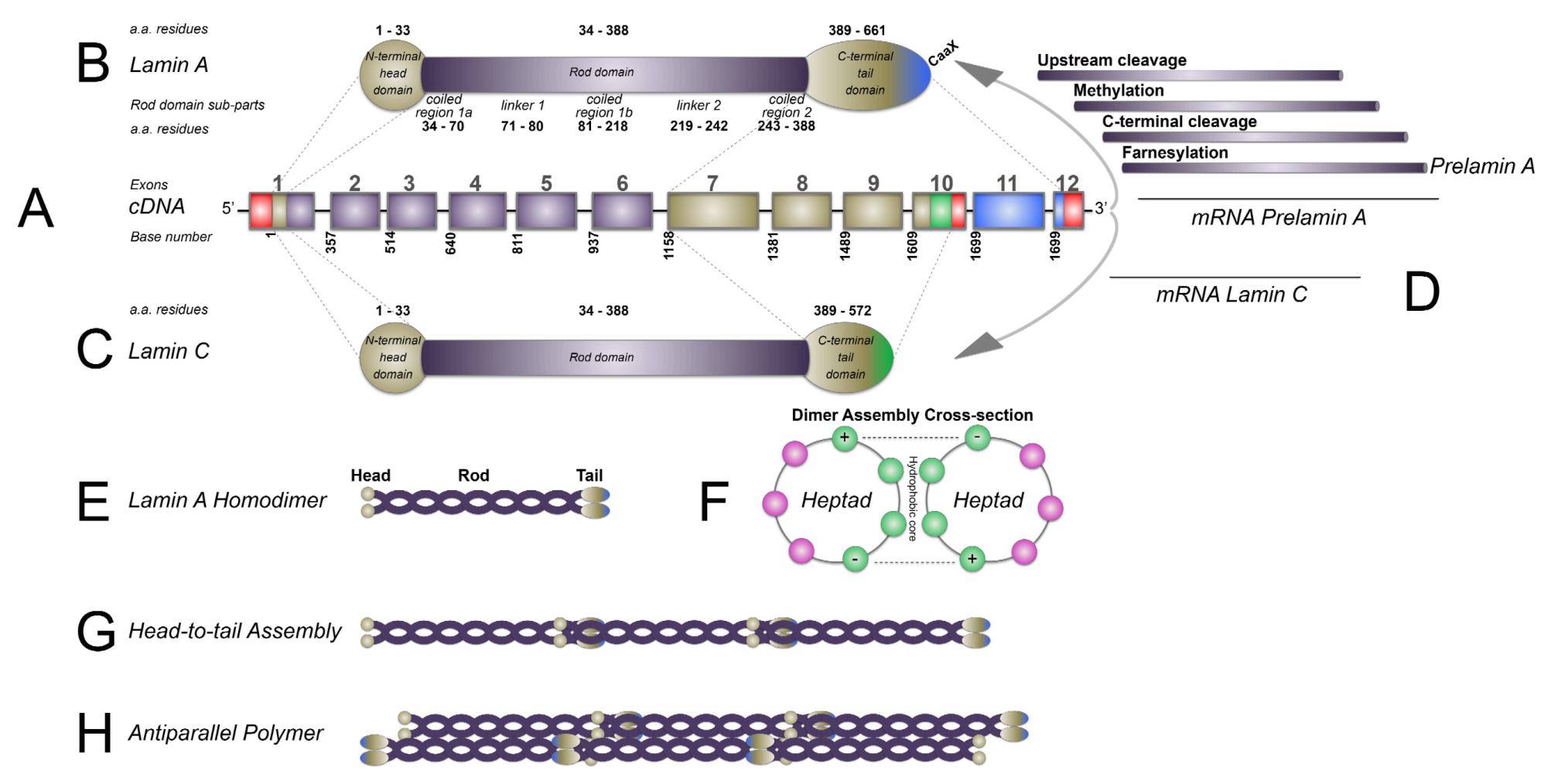


Figure 2. Mutational landscape of LHD-Mutations are spread all over the coding sequence and there is great phenotypic variability.

A Circos of cardiac phenotype (MOGE(S) nosology) against mutation in cDNA order. Each mutation is described in Supplementary Table 1. Exon boundaries are demarcated by sector lines (exon 12 only holds one mutation and the sector is not resolvable). From outside to in: gene mutation variant (text colours indicate mutation type; key, bottom left), then $L M N A$ cDNA location, then structurally and functionally important lamin protein domains (colour key, bottom right), before nested phenotype characteristics per mutation. Internal phenotype tracks contain filled dots collinear to each mutation, which indicate a positive phenotype status as follows: Subclinical = no DCM; the default (not shown) is overt DCM; SA = supraventricular arrhythmia; $\mathrm{CCD}=$ cardiac conduction system disease; eHF = end stage heart failure (heart transplantation or death from eHF); VA = ventricular arrhythmia; $\mathrm{MVA}=$ malignant ventricular arrhythmia (sudden cardiac death, resuscitation or appropriate defibrillator therapy); $\mathrm{CK}=$ elevated creatinine phosphokinase; Juvenile $=$ onset $<25$ years; the default (not shown) is later onset phenotype ( $\geq 25$ years); Msystem $=$ multisystem involvement including neuromuscular, endocrine and lipodystrophy; NYHA = functional class represented as scaled vertical lines (key top right). Circos was constructed in R (package 'Circlize').

DCM, dilated cardiomyopathy; IF-tail, intermediate filament tail. 


\section{NHYA Functional Class}

\section{II , III I IV I}

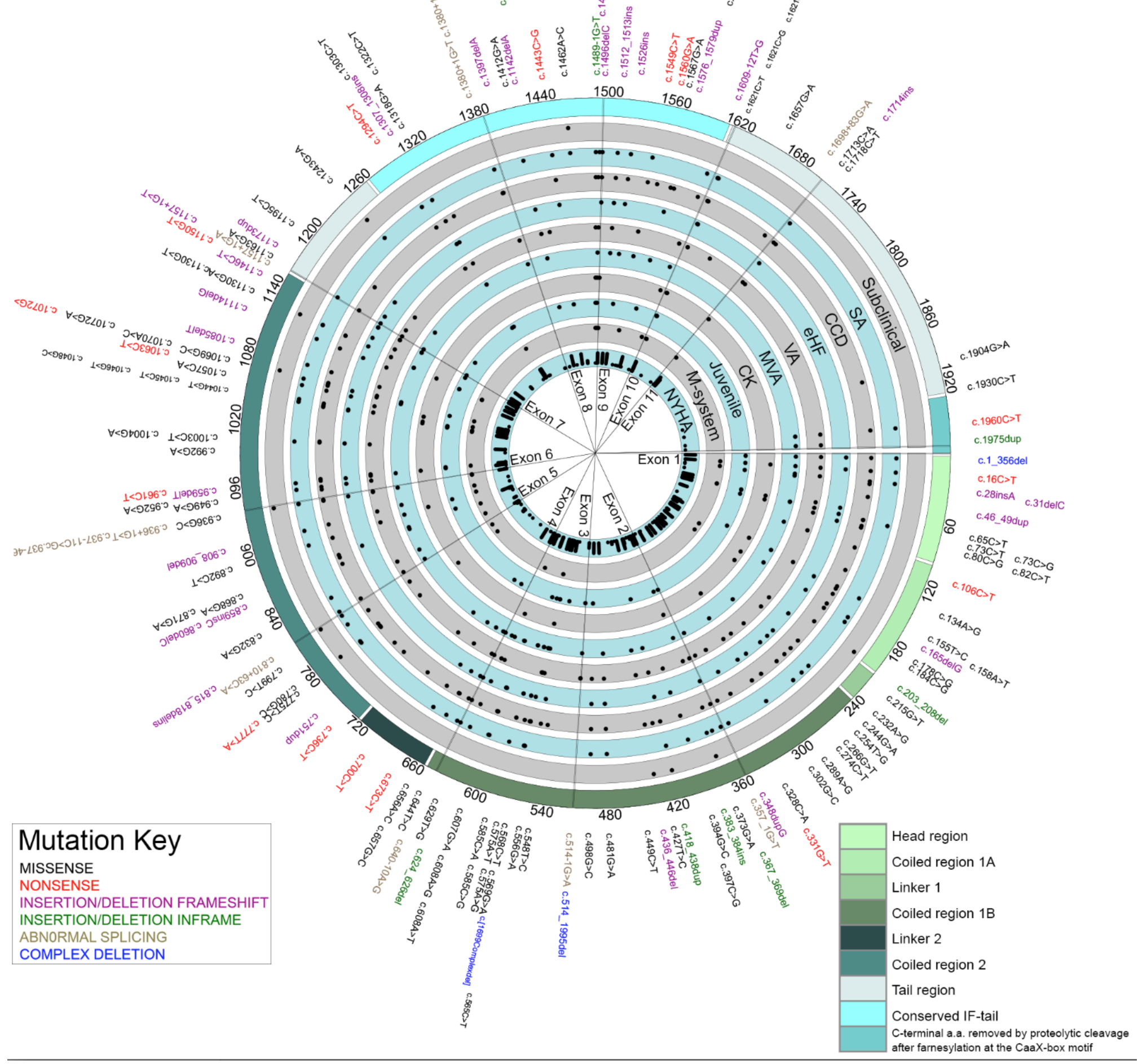


Figure 3. Pathophysiological processes in lamin heart disease-Mutant proteins may cause nuclear instability, altered gene expression, or cell toxicity.

Structural hypothesis (A). A- and B-type lamins form a 3-dimensional molecular scaffold beneath the inner nuclear membrane with extension into the nucleoplasm. Nuclear lamins are coupled to components of the cytoskeleton (cytosolic actin microfilaments, microtubules and cytoplasmic desmin intermediate filaments) by LINC complexes, composed of KASH domain proteins in the outer nuclear membrane and SUN proteins in the inner nuclear membrane. These cytoskeletal networks connect the nuclear lamina to the extracellular matrix, thus maintaining the structural integrity of the whole cell by providing nuclear anchorage, mechanical sensing and structural resistance. LMNA mutations result in impaired myocyte stress resilience, defective force transmission and reduced hypertrophic response to pressure overload.

Gene expression (B). The nuclear envelope plays a role in tissue-specific gene expression that can be altered by $L M N A$ mutations. Lamins interact with nuclear envelope proteins and transcription factors regulating differentiating pathways $\mathrm{Rb} / \mathrm{E} 2 \mathrm{~F}$ or $\mathrm{Rb} / \mathrm{MyoD}$, TGF $\beta$, $\beta$-catenin, and CTGF/CCN2 cascades implicated in cardiac remodelling. They also modulate MAPK and AKT/mTOR pathways through expression of Dusp4. MAPK inhibitors work at 3 levels (yellow cartoons). Because the MAPK pathway through JNK, has also been reported to influence gap junction connexion protein $(\mathrm{Cx} 43)$ expression, dysregulated ion channel trafficking is a potential mechanism for arrhythmogenicity.

Cell toxicity (C). By activating the Akt/mTOR, LMNA mutations impair cardiomyocyte autophagy, making the cell vulnerable to oxidative stress and/or accumulation of toxic misfolded or farnesylated lamins. Temsirolimus facilitates autophagic clearance of mutant protein. Reduced lamin function may also result in SUN1 overexpression, saturation of its nuclear occupancy sites, and its toxic relocation to the Golgi.

AKT, protein kinase B; BAF, barrier to autointegrative factor; DCM, dilated cardiomyopathy; Dusp4, dual specificity phosphatase 4; E2F, E2 factor family of transcription factors; ER, endoplasmic reticulum; ERK1/2, extracellular signal-regulated kinases 1/2; INM, inner nuclear membrane; JNK, c-Jun N-terminal kinases; KASH, Klarsicht, ANC-1, and Syne homology; LAP, Lamin associated protein; LEM, LAP2-emerinMAN1 domain; LINC, linker of the nucleoskeleton and cytoskeleton; MAN1, vertebrate nuclear inner membrane protein; MAPK, mitogen-activated protein kinase; MyoD, myoblast determination protein; mTOR, mammalian target of rapamycin; ONM, outer nuclear membrane; PNS, perinuclear space; R-Smad, $R b$, retinoblastoma protein; SUN, Sad1 and UNC-84 (SUN) proteins; TGF $\beta$, transforming growth factor $\beta$. 

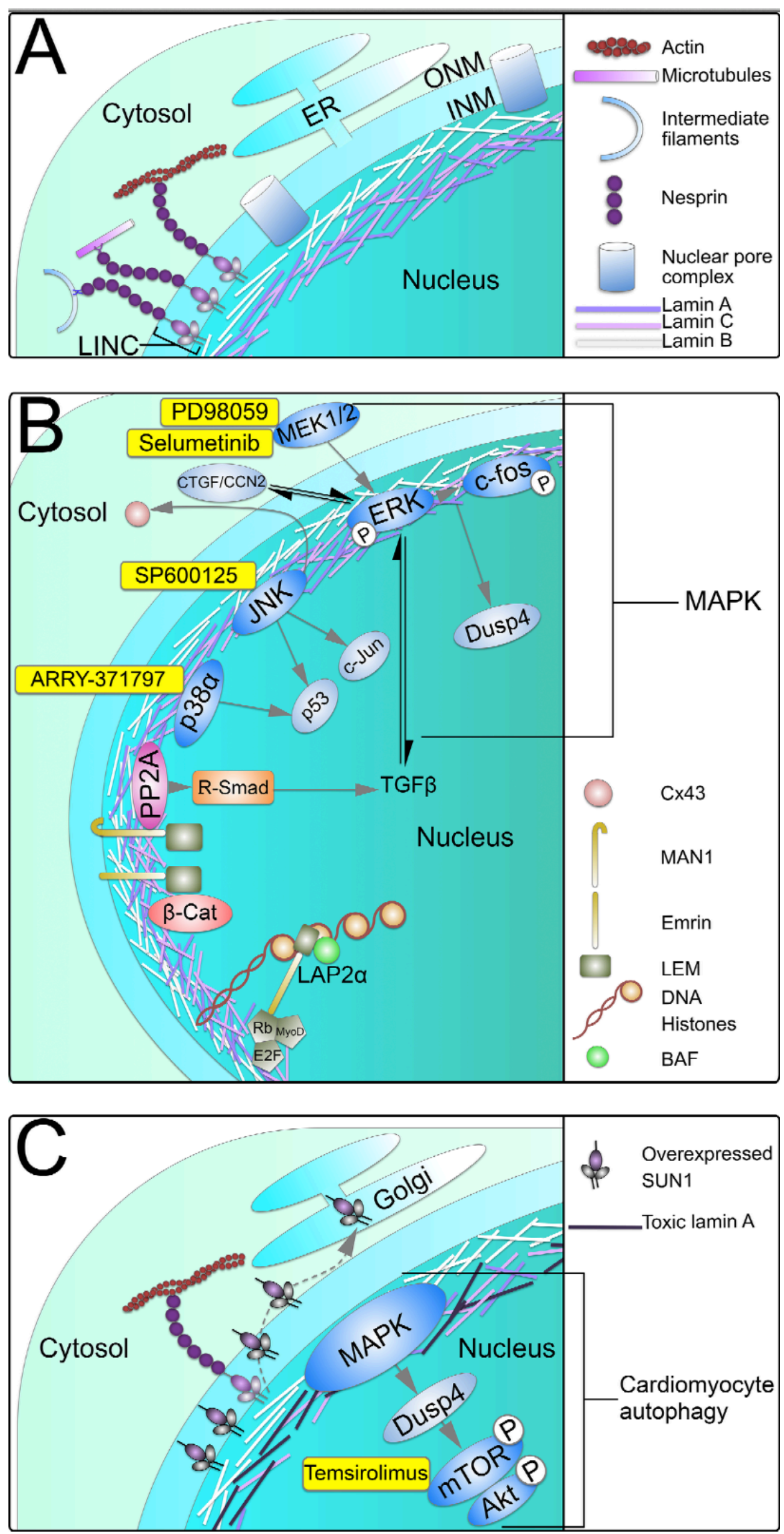
Figure 4. ECG and CMR phenotypes in LHD patients.

ECGs from patients with LHD showing a spectrum of milder electrophysiological phenotypes: A) Borderline first-degree atrioventricular block, RV2>RV3 with fragmented QRS in V3. B) Left bundle branch block. C) Partial right bundle branch block with fragmented QRS in V3. D) RV1>RV2 with fragmented QRS in V2. E) Intraventricular conduction delay, poor R wave progression and QRS fragmentation in V1-3 indicating septal remodelling. F) RV2>RV3 with fragmented QRS in V1. CMR in a 51 Year-old bradycardic male with a pathogenic $L M N A$ mutation and family history of dilated cardiomyopathy and sudden death. Cine imaging (G, H and I) showed a mildly dilated left ventricle of good function (EDV $176 \mathrm{~mL}, \mathrm{EDVi} 103 \mathrm{~mL} / \mathrm{m}^{2}$, ejection fraction 68\%), mildly dilated right ventricle, and biatrial dilatation. Late gadolinium enhancement imaging $(\mathbf{J}, \mathbf{K}, \mathbf{L})$ reveals mid wall non-ischaemic linear fibrosis in many basal segments.

$C M R$, cardiovascular magnetic resonance; ECG, electrocardiogram; EDVi, end-diastolic volume indexed to body surface area; LHD, lamin A/C heart disease.
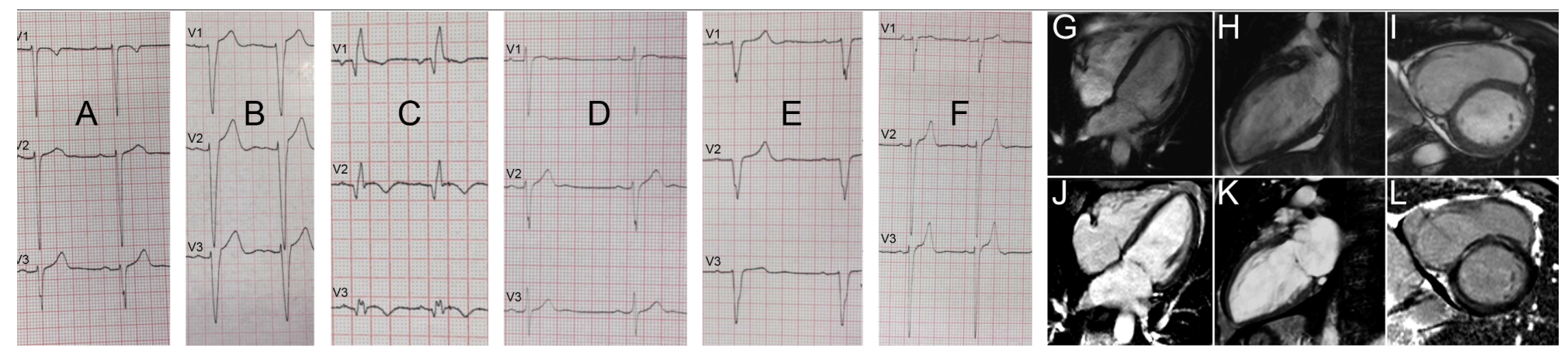


\section{Figure 5. Microscopic phenotype of LHD.}

Pathology (left): Normal lamin A/C immunostain (tan nuclei) in the endomyocardial biopsy of a DCM patient with wildtype $L M N A$ (negative control, $\mathbf{A}$ ). Selective loss and irregularity of lamin A/C expression in myocyte nuclei (red arrows) of patients with these LMNA mutations: p.Glu161Lys (B, C), p.Arg89Leu (D), and p.Arg397Cys (E). Intra-sample positive control provided by the normal nuclear expression of lamin A/C of non-myocyte cells (vascular, endocardial, nervous). Ultrastructural views show extensive nuclear membrane damage (F, red bracket corresponds to nuclear membrane profile) and a combination $(\mathbf{G})$ of nuclear membrane damage (red arrow) and invagination with pore clustering (blue arrow). Fine ultrastructure (right): Recurring nuclear changes in advanced LHD: nuclear membrane convolutions and pseudoinclusions (left images), and intranuclear microtubular proliferation (right images). MOGE(S) descriptor provided per case. $\mathbf{H}, \mathbf{I}$, and $\mathbf{J}$ are from native hearts excised at transplantation.

$A D$, autosomal dominant; $C$, New York Heart Association functional class; $C C D$, cardiac conduction system disease; Ctrl, control; DCM, dilated cardiomyopathy; H, heart. 


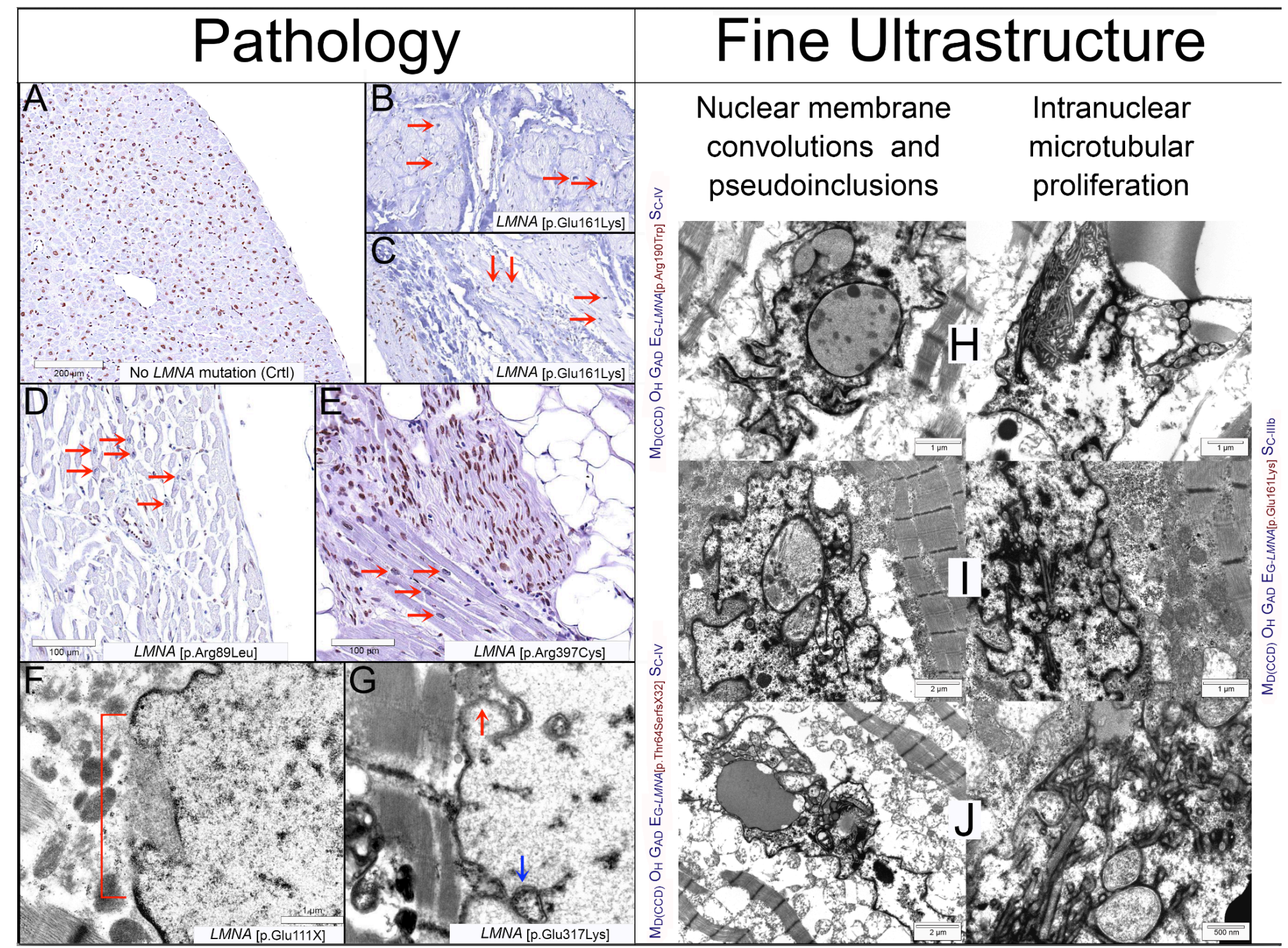


